\title{
GMRT study of $X$-shaped radio sources
}

\section{Dharam Vir Lal ${ }^{* *}$}

Max-Planck-Institut für Radioastronomie, Auf dem Hügel 69, 53121 Bonn, Germany

E-mail: dharamempifr-bonn.mpg.de

\section{A. P. Rao}

National Centre for Radio Astrophysics (TIFR), Pune University Campus, Pune 411007, India

\section{Martin J. Hardcastle}

School of Physics, Astronomy, and Mathematics, University of Hertfordshire, Hatfield, UK

\section{C. Cheung \& Sanjay Bhatnagar}

National Radio Astronomy Observatory, Socorro, P.O. Box 0, New Mexico, USA

\section{Ralph P. Kraft}

Harvard-Smithsonian Center for Astrophysics, MS-67, Cambridge, MA 02138, USA

\section{Andrei P. Lobanov \& Anton J. Zensus}

Max-Planck-Institut für Radioastronomie, Auf dem Hügel 69, 53121 Bonn, Germany

Context. The nature of $X$-shaped sources is a matter of considerable debate in the literature: it has even been proposed that they provide evidence for black-hole-mergers/spin-reorientation, and therefore constrain the rate of strong gravitational wave events.

Aim. To explore the nature of these $X$-shaped radio galaxies.

Method. We conduct a systematic study of a large sample of known and newly discovered $X$ shaped sources along with a comparison sample. We used the Giant Metrewave Radio Telescope with resolution of $\sim 6^{\prime \prime}$ to $\sim 15^{\prime \prime}$ at $610 \mathrm{MHz}$ and $240 \mathrm{MHz}$ in the dual-frequency mode.

Preliminary Result. Based on our careful analysis and estimation of the possible systematic errors, the known $X$-shaped sources divide into the following three categories: (i) the wings have flatter spectral indices than the active lobes, (ii) the wings and the active lobes have comparable spectral indices, and (iii) the wings have steeper spectral indices than the active lobes. In addition, based on our preliminary analysis, one sample source from our comparison sample shows a spectral index result belonging to category (i).

Future. Milliarcsecond scale imaging will be conducted on some of these $X$-shaped sources to investigate if they are examples of binary AGN systems and thereby understand the nature of these sources.

From Planets to Dark Energy: the Modern Radio Universe

October 1-5 2007

The University of Manchester, UK

\footnotetext{
* Speaker.

${ }^{\dagger}$ We thank the staff of the GMRT who have made these observations possible. GMRT is run by the National Centre for Radio Astrophysics of the Tata Institute of Fundamental Research..
} 


\section{Introduction}

A peculiar and small subclass of extragalactic radio sources called $X$-shaped, or 'winged' sources are characterised by two low-surface-brightness lobes (the 'wings') oriented at an angle to the 'active', or high surface brightness radio lobes, giving the total source an ' $X$ ' shape. These two sets of lobes usually pass symmetrically through the centre of the associated host galaxy. Merritt \& Ekers (2002) noted that the majority of these sources are of Fanaroff-Riley type II (FR II) (Fanaroff \& Riley 1974) and the rest are either FR I or mixed.

Formation Scenario Several authors have attempted to explain the unusual structure in $X$-shaped sources. These $X$-shaped radio sources have been put forth as derivatives of central engines that have been reoriented, perhaps due to a minor merger (Merritt \& Ekers 2002; Dennett-Thorpe et al. 2002; Gopal-Krishna et al. 2003). Alternatively, they may also result from two pairs of jets, which are associated with a pair of unresolved AGNs (Lal \& Rao 2005, 2007). These, however, are not the only interpretations for the unusual morphologies; some authors suggest a hydrodynamic origin (Leahy \& Williams 1984; Worrall et al. 1995; Capetti et al. 2002; Kraft et al. 2005) and some suggest a conical precession of the jet axis (Rees 1978; Parma et al. 1985; Mack et al. 1994). See Lal \& Rao (2007) and Cheung (2007) for a detailed account.

\section{Sample \& GMRT Observations}

Known sample The earlier sample of known $X$-shaped sources was drawn from the list mentioned in Merritt \& Ekers (2002) compiled by Leahy \& Parma (1992). These source have been selected solely on the basis of their morphology, and the sample is inhomogeneous and in no sense a statistical complete sample.

Comparison sample The comparison sample consists of all nearby $(z<0.1)$ normal FR II sources from the 3CRR catalogue. These sources have radio luminosities similar to that of the $X$-shaped sources, which lie close to the FR I/FR II divide. We impose an angular-size cutoff (based on high-frequency radio maps) on the target sample and ensure that our sample sources are of similar angular size to typical $X$-shaped sources. In addtion, the sample sources have known weak transverse extensions (proto-wings?) and also have X-ray (XMM/Chandra) observations.

New sample The new sample is drawn from the compiled list of nearly 100 new candidate $X$ shaped radio sources through a search of the FIRST survey database (Cheung 2007). Our sample sources had (i) characteristic ' $X$ ' shape, (ii) both set of lobes passing symmetrically through the centre of the associated host galaxy, and (iii) an angular size of more than $1.2^{\prime}$ as seen in the VLAFIRST 1.4 GHz images.

GMRT Observations The $240 \mathrm{MHz}$ and $610 \mathrm{MHz}$ feeds of GMRT (Swarup et al. 1991) are coaxial feeds and therefore, simultaneous multi-frequency observations at these two frequencies are possible. We made synthesis observations of all our sample sources at $240 \mathrm{MHz}$ and 610 $\mathrm{MHz}$, in the dual frequency mode, using the GMRT during several observing GTAC cycles, in the standard spectral line mode. The GMRT has a hybrid configuration (Swarup et al. 1991) with 14 of its 30 antennas located in a central compact array with size $\sim 1.1 \mathrm{~km}$ and the remaining antennas distributed in a roughly ' $\mathrm{Y}$ ' shaped configuration, giving a maximum baseline length of $\sim 25 \mathrm{~km}$. The hybrid configuration gives reasonably good sensitivity for both compact and extended sources. 


\section{Preliminary Results}

The spectral characteristics of known $X$-shaped sources seem to fall into three distinct categories, namely, sources in which (i) the wings have flatter spectral indices than the active lobes, (ii) the wings and the active lobes have comparable spectral indices, and (ii) the wings have steeper spectral indices than the active lobes. While it is probable that the three categories of sources are unrelated to one another, a single model to explain these sources is a challenge (Lal \& Rao 2007).

We find evidence for the presence of possible unusual spectral properties in typical FR II radio galaxies; i.e., from an independent study of our comparison sample of FR II radio sources, one source from our sample seems to have relatively flatter spectral indices for the low-surfacebrightness features than the high-surface-brightness features.
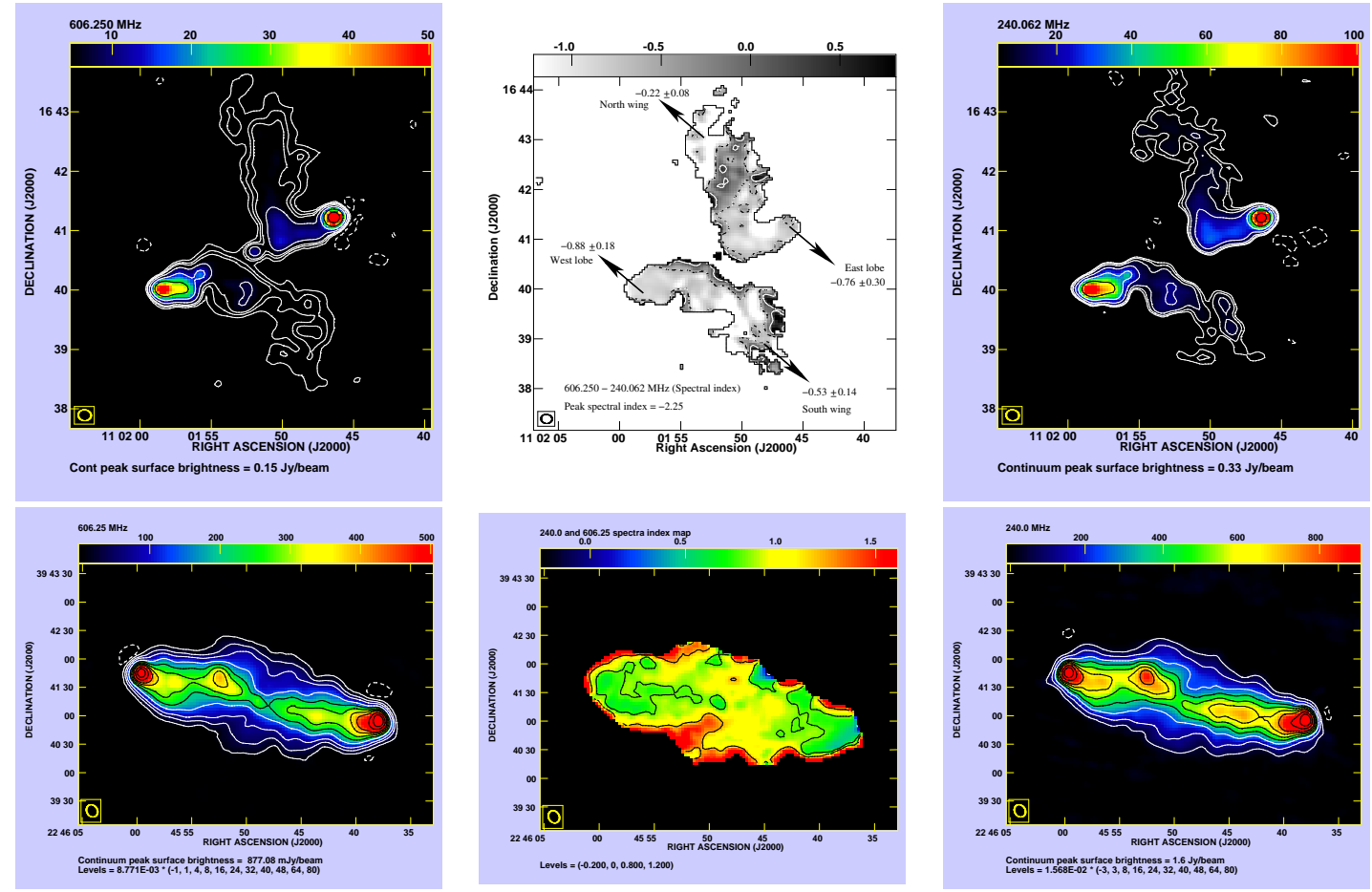

Figure GMRT map of B1059+169 (a known $X$-shaped source) at $610 \mathrm{MHz}$ (top left panel), $240 \mathrm{MHz}$ (top right panel) and the spectral index (240 MHz, $610 \mathrm{MHz}$ ) map (top middle panel). Similarly, the lower panel shows the GMRT map of 4C 452 (a FR II source, possibly missed from the $X$-shaped sample due to projection), from our comparison sample at $610 \mathrm{MHz}$ (bottom left panel), $240 \mathrm{MHz}$ (bottom right panel) and the spectral index (240 MHz, $610 \mathrm{MHz}$ ) map (bottom middle panel). The CLEAN beams for $610 \mathrm{MHz}$ and $240 \mathrm{MHz}$ maps are $\sim 6^{\prime \prime}$ and $\sim 15^{\prime \prime}$, respectively. The GMRT map at $610 \mathrm{MHz}$ is matched with the resolution of $240 \mathrm{MHz}$. Note the unusual spectral index in B1059+169.

\section{References}

[1] Kraft, R.P., Hardcastle, M.J., Worrall, D.M. \& Murray, S.S. 2005, ApJ, 622, 149

[2] Lal, D.V. \& Rao, A.P. 2007, MNRAS, 374, 1085

[3] Merritt, D. \& Ekers, R.D. 2002, Sci, 297, 1310 